\title{
The Brezis-Nirenberg problem for fractional systems with Hardy potentials
}

\author{
Yansheng Shen ${ }^{1}$ \\ ${ }^{1}$ Beijing Normal University
}

March 4, 2021

\begin{abstract}
In this work we study the existence of positive solutions to the following fractional elliptic systems with Hardy-type singular potentials, and coupled by critical homogeneous nonlinearities $\backslash$ begin $\left\{\right.$ equation*\} $\backslash$ begin $\{$ cases $\}(-\backslash \text { Delta })^{\wedge}\{\mathrm{s}\} \mathrm{u}_{-} \backslash \mathrm{mu} \mathrm{m}_{-}$ $\{1\} \backslash \operatorname{frac}\{\mathrm{u}\}\left\{|\mathrm{x}|^{\wedge}\{2 \mathrm{~s}\}\right\}=|\mathrm{u}|^{\wedge}\left\{2^{\wedge}\{\backslash \text { ast }\}_{-}\{\mathrm{s}\}-2\right\} \mathrm{u}+\backslash$ frac $\{\backslash$ eta $\backslash$ alpha $\}\left\{2^{\wedge}\{\backslash \text { ast }\}_{-}\{\mathrm{s}\}\right\}|\mathrm{u}|^{\wedge}\{\backslash$ alpha- 2$\}|\mathrm{v}|^{\wedge}\{\backslash$ beta $\} \mathrm{u}+\backslash$ frac $\{1\}\{2\} \mathrm{Q}_{-}\{\mathrm{u}\}(\mathrm{u}, \mathrm{v})$ $\backslash \backslash$ in $\backslash \backslash$ Omega, $\backslash \backslash[2 \mathrm{~mm}](-\backslash \text { Delta })^{\wedge}\{\mathrm{s}\} \mathrm{v}_{-} \backslash \operatorname{mu}_{-}\{2\} \backslash$ frac $\{\mathrm{v}\}\left\{|\mathrm{x}|^{\wedge}\{2 \mathrm{~s}\}\right\}=|\mathrm{v}|^{\wedge}\left\{2^{\wedge}\{\backslash \text { ast }\}_{-}\{\mathrm{s}\}-2\right\} \mathrm{v}+\backslash$ frac $\{\backslash$ eta $\backslash$ beta $\}\left\{2^{\wedge}\{\backslash\right.$ ast $\left.\}-\{\mathrm{s}\}\right\}|\mathrm{u}|^{\wedge}\{\backslash$ alpha $\}$ $|\mathrm{v}|^{\wedge}\{\backslash$ beta-2 $\} \mathrm{v}+\backslash \operatorname{frac}\{1\}\{2\} \mathrm{Q}_{-}\{\mathrm{v}\}(\mathrm{u}, \mathrm{v}) \backslash \backslash$ in $\backslash \backslash$ Omega, $\backslash \backslash[2 \mathrm{~mm}] \backslash \backslash \mathrm{u}, \backslash \mathrm{v}>0 \backslash \backslash \backslash \backslash \backslash$ in $\backslash \backslash \backslash$ Omega, $\backslash \backslash[2 \mathrm{~mm}] \backslash \mathrm{u}=\mathrm{v}=0 \backslash \backslash \backslash \backslash$ in $\backslash \backslash \backslash \operatorname{mathbb}\{\mathrm{R}\}^{\wedge}\{\mathrm{N}\} \backslash$ backslash $\backslash$ Omega, $\backslash$ end $\{$ cases $\} \backslash$ end $\{$ equation* $\}$ where $\$(-\backslash \text { Delta })^{\wedge}\{\mathrm{s}\} \$$ denotes the fractional Laplace operator, $\$ \backslash$ Omega $\backslash$ subset $\backslash$ mathbb $\{\mathrm{R}\}^{\wedge}\{\mathrm{N}\} \$$ is a smooth bounded domain such that $\$ 0 \backslash$ in $\backslash$ Omega $\$, \$ \backslash \operatorname{mu}_{-}\{1\}, \backslash$ mu_ $\{2\} \backslash$ in $\left[0, \backslash \operatorname{Lambda}_{-}\{\mathrm{N}, \mathrm{s}\}\right) \$, \$ \backslash \operatorname{Lambda}_{-}\{\mathrm{N}, \mathrm{s}\}=2^{\wedge}\{2 \mathrm{~s}\} \backslash \operatorname{frac}\left\{\backslash \operatorname{Gamma}^{\wedge}\{2\}(\backslash \operatorname{frac}\{\mathrm{N}+2 \mathrm{~s}\}\{4\})\right\}\left\{\backslash\right.$ Gamma $^{\wedge}\{2\}(\backslash$ frac $\left.\{\mathrm{N}-2 \mathrm{~s}\}\{4\})\right\} \$$ is the best constant of the fractional Hardy inequality and $\$ 2^{\wedge}\left\{{ }^{*}\right\}_{-}\{\mathrm{s}\}=\backslash$ frac $\{2 \mathrm{~N}\}\{\mathrm{N}-2 \mathrm{~s}\} \$$ is the fractional critical Sobolev exponent. In order to prove the main result, we establish some refined estimates on the extremal functions of the fractional Hardy-Sobolev type inequalities and we get the existence of positive solutions to the systems through variational methods.
\end{abstract}

\section{Hosted file}

Fractional-system2021.pdf available at https ://authorea.com/users/399485/articles/512008-thebrezis-nirenberg-problem-for-fractional-systems-with-hardy-potentials

\section{Hosted file}

Fractional-system2021. tex available at https://authorea.com/users/399485/articles/512008-thebrezis-nirenberg-problem-for-fractional-systems-with-hardy-potentials 